\title{
The potential utilizations of hydrogen as a promising therapeutic strategy against ocular diseases
}

\author{
This article was published in the following Dove Press journal: \\ Therapeutics and Clinical Risk Management \\ 19 May 2016 \\ Number of times this article has been viewed
}

\author{
Ye Tao ${ }^{1, *}$ \\ Lei Geng',* \\ Wei-Wei Xu' \\ Li-Min Qin' \\ Guang-Hua Peng' \\ Yi-Fei Huang' \\ 'Department of Ophthalmology, \\ ${ }^{2}$ Department of Orthopaedics, \\ Chinese People's Liberation Army \\ General Hospital, Ophthalmology \& \\ Visual Science Key Lab of PLA, Beijing, \\ People's Republic of China \\ *These authors contributed equally \\ to this work
}

Correspondence: Yi-Fei Huang;

Guang-Hua Peng

Department of Ophthalmology, Chinese

People's Liberation Army General

Hospital, 28 Fuxing Road, Haidian

District, Beijing 100853, People's

Republic of China

Tel +86 I0 $66937943 ;+861066937949$

Fax +86 I0 $68286682 ;+861068286637$

Email huangyf30I@I63.com;

peng63088@I63.com

\begin{abstract}
Hydrogen, one of the most well-known natural molecules, has been used in numerous medical applications owing to its ability to selectively neutralize cytotoxic reactive oxygen species and ameliorate hazardous inflammations. Hydrogen can exert protective effects on various reactive oxygen species-related diseases, including the transplantation-induced intestinal graft injury, chronic inflammation, ischemia-reperfusion injuries, and so on. Especially in the eye, hydrogen has been used to counteract multiple ocular pathologies in the ophthalmological models. Herein, the ophthalmological utilizations of hydrogen are systematically reviewed and the underlying mechanisms of hydrogen-induced beneficial effects are discussed. It is our hope that the protective effects of hydrogen, as evidenced by these pioneering studies, would enrich our pharmacological knowledge about this natural element and cast light into the discovery of a novel therapeutic strategy against ocular diseases.
\end{abstract}

Keywords: hydrogen, therapeutic strategy, ocular diseases

\section{Introduction}

Reactive oxygen species (ROS) is a group of highly reactive molecules that are generated during energy-generating biochemical reactions and cellular functions. ${ }^{1-3}$ Among them, physiological ROS are recognized as necessary signaling molecules in their own right. They are capable of oxidizing the phosphatases, kinases, as well as other vital proteins, and thereby efficiently modulate the metabolism and immune system. ${ }^{4}$ Nevertheless, cytotoxic ROS subtypes, such as the hydroxyl radicals $(\cdot \mathrm{OH})$ and the peroxynitrite $\left(\mathrm{ONOO}^{-}\right)$, can break down the mitochondrial membrane and promote the release of cytochrome-c, which further activates the downstream apoptotic cascade. ${ }^{5}$ Thus timely elimination of these cytotoxic ROS is necessary without compromising other physiologically beneficial ROS, which are essentially correlated with the biological activities.

Generally, the enzymatic and nonenzymatic antioxidants build up an endogenous antioxidant defense to neutralize the cytotoxic ROS selectively. However, if the oxidative stress successively accumulates in the eyes and the protection from endogenous antioxidants is insufficient to neutralize the surplus ROS or maintain the cellular homeostasis, these excessive ROS would result in detrimental effects and ultimately cause cell death. ${ }^{6,7}$ Hitherto, accumulating evidence suggests that the etiology of multiple ocular diseases, including cataract, glaucoma, age-related macular degeneration, retinitis pigmentosa, diabetic retinopathy (DR), and multiple traumatic injuries, ${ }^{8}$ are closely related to ROS. Therefore, it is necessary and reasonable to supply exogenous antioxidants to suppress the progression of ocular pathologies and alleviate the eyes from oxidative injury (Figure 1). The beneficial effects induced by 


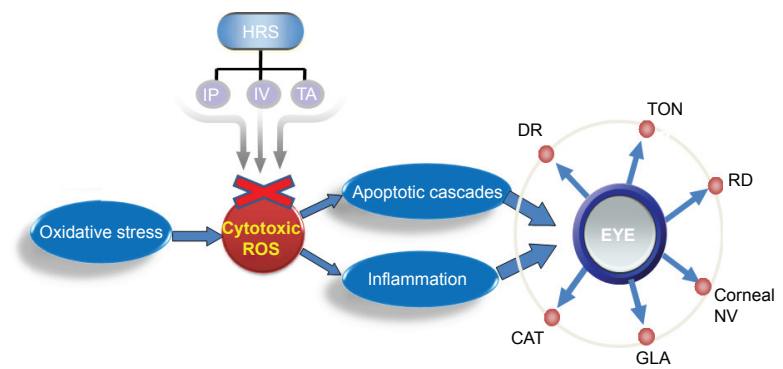

Figure I The basic principles of hydrogen-induced therapeutic effects against ophthalmological pathologies.

Abbreviations: CAT, cataract; Corneal NV, corneal neovascularization; DR, diabetic retinopathy; GLA, glaucoma; HRS, hydrogen-rich saline; IP, intraperitoneal; IV, intravitreous; RD, retinal degeneration; ROS, reactive oxygen species; TA, topical administration; TON, traumatic optic neuropathy.

the exogenous antioxidants to ameliorate ocular diseases are exciting. ${ }^{9,10}$ However, several barriers can impede the therapeutic utilization of these exogenous scavengers, such as the low membrane permeability and the high toxic side effects. Furthermore, these disadvantages would constrain the administration of these therapies to a narrow window of therapeutic dosage. ${ }^{11}$

Hydrogen, a colorless, tasteless, and odorless diatomic gas, was initially recognized as a medical therapeutic substance in $1975 .{ }^{12}$ Subsequently, Ohsawa et al ${ }^{13}$ identified hydrogen-induced protective effects on a rat model of cerebral infarction in 2007 and highlighted the discovery of a gaseous radical scavenger. Thereafter, hydrogen-induced therapeutic effects on a wide range of diseases have been reported, including transplantation-induced intestinal graft injuries, cognitive deficits, inflammatory diseases, Parkinson's disease, metabolic syndromes, as well as ischemia-reperfusion (I/R) injury in the brain, liver, myocardium, intestines, and kidneys. ${ }^{14-22}$

Hydrogen can exert antioxidant and antiapoptotic activities via selectively neutralizing the cytotoxic ROS, such as the $\cdot \mathrm{OH}$ and $\mathrm{ONOO}-$. Meanwhile, the signaling ROS playing metabolic roles, such as the superoxide and hydrogen peroxide, are far less affected. ${ }^{13}$ Unlike some antioxidant supplements with strong reductive reactivity, hydrogen neither disturbs the physiological oxidation reactions nor disrupts the essential defensive mechanisms. Recently, it has been verified that hydrogen can upregulate the expression levels of several endogenous antioxidant enzymes, including superoxide dismutase, glutathione peroxidase, glutathione reductase, and glutathione transferase. ${ }^{23,24}$ These advantages indicate that the application of hydrogen therapy might act as a safe and effective strategy against ROS-related disorders without giving rise to serious side effects.
Hydrogen can penetrate the membranes and diffuse into organelles (eg, the mitochondria and nuclear DNA), and thereby get access to the intracellular source of cytotoxic ROS. ${ }^{13,24}$ Based on the favorable distribution characteristic, hydrogen is highly effective for reducing the level of cytotoxic ROS, which impair the nuclear DNA and mitochondria. It has been found that inhalation of hydrogen is more effective than edaravone, an approved ROS scavenger in Japan, in alleviating the effects of ischemia-induced oxidative injury in neurons. ${ }^{25}$ Furthermore, hydrogen is considered an antiinflammatory because it significantly reduces the circulating levels of multiple proinflammatory cytokines, such as the chemokine ( $\mathrm{C}-\mathrm{C}$ motif), ligand 2 (CCL2), interleukin (IL) $-1 \beta$, IL-6, and tumor necrosis factor- $\alpha .{ }^{26-28}$ Particularly, hydrogen can ameliorate the accumulation of activated microglia, which indicates inflammation and remodeling. ${ }^{29}$

Generally, hydrogen can be administered in a straightforward way by providing the gas for the subject to inhale using a ventilator circuit, facemask, or nasal cannula. ${ }^{30-32}$ A subsequent study suggests that hydrogen can be dissolved in water up to $0.8 \mathrm{mM}$ under atmospheric pressure at room temperature and its solubilized form, the hydrogen-rich saline (HRS), is advantageous since it is a safe, portable, and easily handled approach for delivery. ${ }^{24,33,34}$ More importantly, a higher concentration of hydrogen can be dissolved and comprised in the HRS. Thus far, both in vitro and in vivo studies have verified that the antioxidant properties of HRS can decrease the incidence of ROS-related diseases. HRS can be administered orally, or in the form of peritoneal or intravenous injections. ${ }^{35-37}$ Particularly, in the eye, it can be delivered by topical drops or intravitreal injections. ${ }^{38,39}$

The basic design and goal of potential clinical practice is to provide safe hydrogen delivery and precise efficiency analysis. The tissue compatibility of hydrogen is satisfactory because it is an endogenous substance, which is continuously produced in the human intestine.${ }^{40}$ From the aspect of safety, hydrogen is advantageous to many other antioxidants as it shows no cytotoxicity even at high concentrations. Inhalation of hydrogen has already been used in the prevention of decompression sickness in divers and provided good safety profiles. ${ }^{30}$

\section{The potential utilization of hydrogen against ocular diseases}

Hydrogen can modulate several biological functions, and exhibits antioxidant and anti-inflammatory effects. The ability of hydrogen to neutralize free radicals, especially the hydroxyl radicals as well as other detrimental ROS, can 


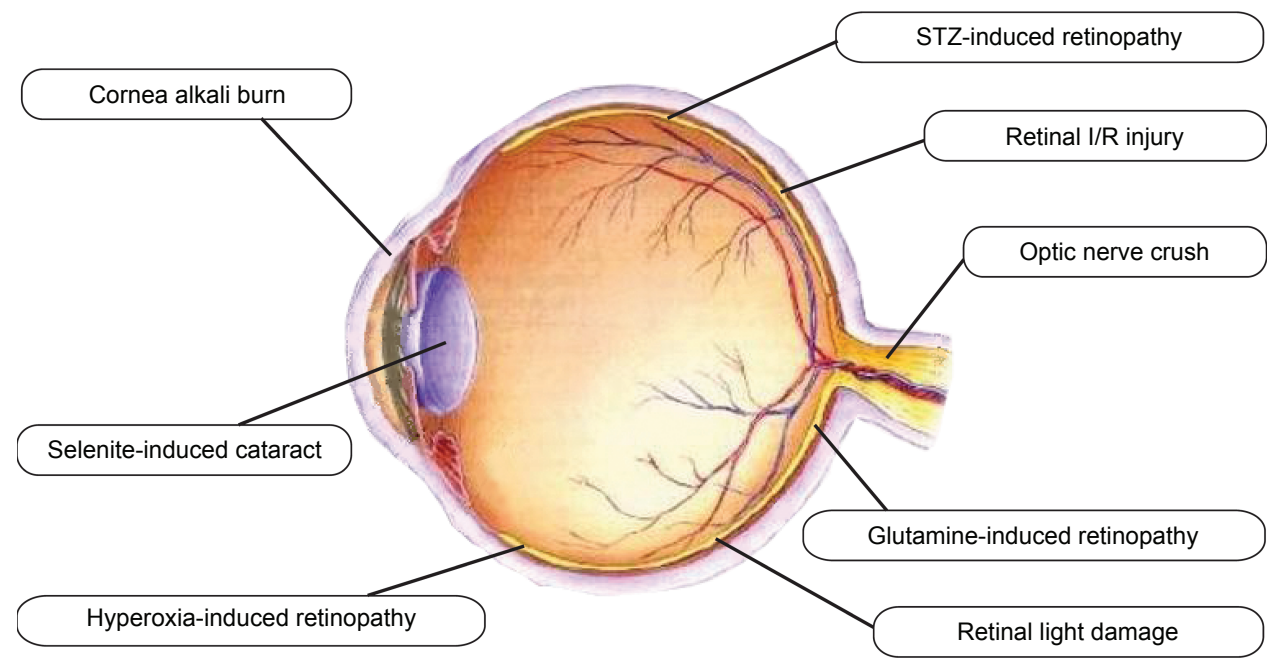

Figure 2 The novel utilizations of hydrogen against multiple ophthalmological pathologies.

Abbreviations: I/R, ischemia-reperfusion; STZ, streptozotocin.

be utilized to treat or prevent ocular disorders related to oxidative stress. In clinical practice, ophthalmologic utilization of hydrogen remains a rarely touched field. However, a series of pioneering experiments based on laboratory evidences have verified the valuable opportunities to develop hydrogen into a therapeutic tool against ocular diseases (Figure 2). Herein, we summarize the recent utilizations of hydrogen in the ophthalmological experiments and discuss its potential clinical feasibility (Table 1).

\section{The potential therapeutic effects against retinal degeneration}

Retinal degeneration (RD) is a heterogeneous group of inherited retinal dystrophies that characterized by the progressive photoreceptor apoptosis. ${ }^{41-43}$ Current therapeutic strategies against RD include the gene therapy, nutritional supplements, antiapoptotic therapy, retinal transplantation, retinal prostheses, and stem cell therapy. However, the therapeutic effects of the aforementioned approaches remain unsatisfactory, owing to the complexity of etiology and the chronic cycle of pathological progression. ${ }^{44,45}$ It has been shown that the ROS plays a pivotal role in caspase-independent photoreceptor apoptosis in RD. ${ }^{46}$ This notion is further supported by the fact that multiple antioxidants have been successful in effectively suppressing photoreceptor degeneration in RD models. ${ }^{47-49}$ Given the potent scavenging ability of hydrogen, it is reasonable to hypothesize that exogenous supplementation of hydrogen can ameliorate oxidative stress in RD and act as a therapeutic strategy to retard or prevent the photoreceptor degeneration.

Excessive exposure to light can induce the formation and accumulation of ROS in retinas and eventually results in the photoreceptors' apoptosis. ${ }^{43,49}$ The pathologic mechanism of the light-induced RD is partly similar to that of RD.

Table I Novel experimental investigations of hydrogen against the ocular pathologies (up to December 20I5)

\begin{tabular}{|c|c|c|c|c|}
\hline Animals & Disease models & Human diseases & HRS Delivery approach & Time and investigator \\
\hline Rat & Retinal light damage & RD & IP injection & Tian et $\mathrm{al}^{51}$ \\
\hline Rat & Retinal light damage & RD & IP injection & Feng et $\mathrm{al}^{50}$ \\
\hline Rat & STZ induced retinopathy & DR & IP injection & Xiao et $\mathrm{al}^{56}$ \\
\hline Rat & STZ induced retinopathy & DR & IP injection & Feng et $\mathrm{al}^{57}$ \\
\hline Mouse & Hyperoxia induced retinopathy & DR & IP injection & Huang et $\mathrm{al}^{60}$ \\
\hline Rat & Optic nerve crush & TON & IP injection & Sun et $\mathrm{al}^{64}$ \\
\hline Rat & Selenite induce cataract & Cataract & IP injection & Yang et $\mathrm{al}^{23}$ \\
\hline Rat & Retinal I/R injury & Glaucoma & Topical drops & Oharazawa et $\mathrm{al}^{39}$ \\
\hline Rat & Retinal I/R injury & Glaucoma & IP injection & Liu et $\mathrm{al}^{77}$ \\
\hline Guinea pig & Glutamine induced retinopathy & Glaucoma & IP and IV injection & Wei et $\mathrm{al}^{38}$ \\
\hline Mouse & Cornea alkali-burn & Corneal neovascularization & Topical irrigation & Kubota et $\mathrm{al}^{71}$ \\
\hline
\end{tabular}

Abbreviations: HRS, hydrogen rich saline; RD, retinal degeneration; DR, diabetic retinopathy; TON, traumatic optic neuropathy; IP, intraperitoneal injection; IV, intravitreous injection; STZ, streptozotocin. 
Therefore, this highly reproducible model has been widely utilized in pathologic and therapeutic investigation of human RD. Hitherto, two independent studies have found that saturated hydrogen saline can protect the retina from lightinduced damage by attenuating the oxidative stress. ${ }^{50,51}$ It was shown that both retinal architecture and dark adapted electroretinogram (ERG) forms were relatively intact in hydrogen-treated rats compared with the vehicle-treated controls. ${ }^{51}$ In greater detail, investigations using the electron microscope found that the hydrogen treatment could protect the cellular organelles of the photo receptors against the light induced injury. To some extent, these microstructure results verify that hydrogen can penetrate the membranes and then enter the nucleus and mitochondria. Moreover, the retinal malondialdehyde concentration, a presumptive marker of lipid peroxidation, was significantly reduced by hydrogen therapy, indicating that the protective effects of hydrogen could be ascribed to its antioxidant properties. ${ }^{50}$ These encouraging findings based on the phototoxic models verify the efficacy of hydrogen to arrest photoreceptor degeneration and cast light on the discovery of a novel therapeutic to prevent the retinal damages in age-related macular degeneration or retinitis pigmentosa. From a long-term perspective, delaying the occurrence and progression of RD by clinical hydrogen therapy and establishing an efficient administrative protocol are the focus of future research.

\section{The potential therapeutic effects against DR}

DR is the leading cause of blindness in the working force of developed countries and also acts as a significant cause of blindness in the elderly population. ${ }^{52}$ The overall prevalence of retinopathy among patients with diabetes is around $26 \%$. Both clinical and experimental studies have demonstrated that DR is closely related to oxidative stress: the ROSinduced biochemical changes can induce both structural and functional abnormalities in the microvasculature of diabetic retinas, resulting in the breakdown of blood-retinal barrier. ${ }^{53,54}$ The blood-retinal barrier is critical for partitioning the neural elements of the retina from the circulation and protecting the retina from circulating inflammatory cells and cytotoxic products. ${ }^{55}$ Consequently, ROS has been considered as a major contributor to the pathology of DR. A novel research sought to explore the effect of HRS on the streptozotocin-induced DR rat model. It was found that HRS can suppress caspase activity, ameliorate retinal apoptosis, and reduce vascular permeability. ${ }^{56}$ Moreover, the HRS therapy can attenuate retinal parenchyma thickening. Another study also adopted HRS to alleviate the streptozotocin-induced DR and found that the blood-retinal barrier in these treated rats was effectively preserved. ${ }^{57}$

The ROS could upregulate the expression levels of vascular endothelial growth factor (VEGF) and enhance VEGF receptor activity via complex signaling networks. ${ }^{58,59}$ VEGF can give rise to choroidal neovascularization (CNV) in the DR and has been considered as a therapeutic target in clinical practice. A pioneering study based on the hyperoxia-induced mouse model found that HRS can counteract the $\mathrm{CNV}$ and reduce VEGF expression via inhibition of oxidative stress. ${ }^{60}$ Therefore, the anti-VEGF property should be considered as another functional factor for hydrogeninduced protection. These preliminary studies indicate that hydrogen might act as a valid candidate in further clinical treatment of DR. Although the possible effect of hydrogen has not been clinically verified in DR patients hitherto, a randomized, double-blind, placebo-controlled, crossover study has already found that the supplementation of HRS improved lipid and glucose metabolism in patients with type 2 diabetes or impaired glucose tolerance. ${ }^{61}$ These hydrogeninduced effects might be potentially applied to the diabetic complications, especially to DR, in which metabolic disorder plays a pivotal role.

\section{The potential therapeutic effects against traumatic optic neuropathy}

Traumatic optic neuropathy is one of the most devastating causes of permanent visual loss and complete blindness. After traumatic injury, such as the optic nerve crush, prominent signs of retinal ganglion cells (RGCs) apoptosis are found in the injured retinas. ${ }^{62}$ Oxidative stress is now considered a pathologic factor that mediates the post-trauma process of RGCs apoptosis. ${ }^{63}$ A pioneering study found that hydrogen can promote the survival of RGCs and enhance visual function recovery in an optic nerve crush rat model. ${ }^{64}$ Moreover, the terminal deoxynucleotidyl transferase dUTP nick end labeling staining and malondialdehyde assay suggested that the neuroprotective effects were mediated by suppressing the ROS-induced apoptosis. These instrumental findings highlight the possibility of developing hydrogen into an effective tool against the traumatic optic neuropathy in clinical practice.

\section{The potential therapeutic effects against cataract}

Cataract is the leading cause of blindness around the world and affects up to $80 \%$ of the human population over the age of 
70 years. ${ }^{65}$ Its prevalence is increasing in many countries due to the growth of aged population. Currently, the only effective treatment for cataract is surgical removal and replacement of the cataract with an artificial intraocular lens. ${ }^{66}$ Nevertheless, an initial study suggested that hydrogen might act as an alternative therapy for the cataract. ${ }^{23}$ It has been shown that hydrogen can retard cataract formation and restore the antioxidant capacity in a selenite cataract model by maintaining the activities of multiple enzymatic and nonenzymatic antioxidants. Malondialdehyde accumulation in the lenses of a selenite-treated rat was also efficiently suppressed, indicating that hydrogen possibly prevented the oxidation of lenticular membrane lipids. According to the classic theories, ROS is a crucial etiologic contributor to the initiation of cataract: ROS leads to a surge of detrimental biochemical reactions in the lens, including crosslinking and aggregation of lens proteins, peroxidation of membrane lipids, apoptosis of epithelial cells of the lens, and the eventual formation and progression of cataract. ${ }^{67}$ In view of the potent ROS scavenging ability of hydrogen, it is reasonable to further explore its therapeutic effects for cataract pathology in clinical settings.

\section{The potential therapeutic effects against alkali injury of cornea}

Accidental alkali burn is a terrible trauma to the eye that results in acute inflammation and secondary pathologic corneal neovascularization in the cornea. The balance between angiogenic and antiangiogenic factors determines the fate of corneal neovascularization as well as prognostic vision. ${ }^{68,69}$ Thus far, existing therapies to prevent the onset of corneal neovascularization, such as the antiangiogenic drugs, argon laser photocoagulation, photodynamic therapy, and limbal stem cell transplantation et.al, are far from effective and efficient. ROS can activate the transcription nuclear factor kappa B (NF-kB), which then translocates to the nucleus to induce the expression of inflammatory cytokines such as VEGF, monocyte chemotactic protein-1 (MCP-1), IL, and tumor necrosis factor- $\alpha .^{70}$ These cytokines not only induce corneal neovascularization, but can also recruit inflammatory cells that further exacerbate inflammation. Therefore, ROS should be considered a key trigger for pathologic corneal neovascularization and inflammatory response in alkali-burned cornea. This hypothesis has been corroborated by an animal study: ROS can directly give rise to pathologic corneal neovascularization through the activation of the NF-kB pathway in the mouse cornea after an alkali-burn injury. ${ }^{71}$ More importantly, the topical use of hydrogen-enriched solution can significantly reduce angiogenesis in these corneas. It was found that hydrogen water irrigation significantly quenched NF-kB phosphorylation and abated the VEGF protein level. These encouraging findings suggest that the oxidative stress at the onset of corneal alkali injury can be an upstream target for therapeutic intervention. Hydrogen might be developed into a potent treatment against angiogenesis in alkali-burned corneas.

\section{The potential therapeutic effects against glaucoma}

Transient elevation of intraocular pressure is known to induce retinal $\mathrm{I} / \mathrm{R}$ injury and result in the necrosis and apoptosis of retinal neurons. ${ }^{72,73}$ These pathological features closely resemble those of acute angle-closure glaucoma. The underlying mechanisms of $I / R$ injury are closely related to the formation of ROS, which has been recognized as a contributor to the pathogenesis of glaucomatous neurodegeneration. ${ }^{74}$ Encouragingly, it has been shown that the endogenous supplementation with antioxidant enzymes or ROS scavengers can retard or prevent I/R injuries in many mammal retinas. ${ }^{75,76} \mathrm{In}$ a recent study based on the rat model, continuous administration of hydrogen-loaded eye drops immediately increased the hydrogen concentration in the vitreous body and suppressed the $\mathrm{I} / \mathrm{R}$-induced oxidative stress, leading to a decrease in retinal neuron apoptosis. ${ }^{39}$ Moreover, it has been verified that hydrogen treatment inhibits the activation of microglia, which could give rise to ongoing neurodegeneration in the injured retinas. Another study sought to explore the anti-apoptosis mechanism of HRS therapy found that HRS alleviated retinal I/R injury through the inhibition of poly ADP-ribose polymerase 1 , a nuclear enzyme involved in the regulation of multiple pathophysiological cellular procedures, including DNA oxidation and caspase-3-mediated apoptosis. ${ }^{77}$ These antiapoptotic and anti-inflammatory properties would inspire us to test the therapeutic effects of hydrogen against the glaucomatous pathologies in future clinical work.

Glutamate-induced excitotoxicity is another intraocular pressure-independent factor contributing to the apoptosis of RGCs in glaucoma. ${ }^{78}$ Administration of HRS reduced glutamate excitotoxic injury and enhanced retinal recovery in guinea pigs. ${ }^{38}$ These beneficial results could be ascribed to the suppression of the glial cells and the promotion of glutamate clearance. These underlying mechanisms will enrich our knowledge of hydrogen as a novel therapeutic element against glaucoma.

\section{Conclusion}

Therapeutic medical gas can act as a reasonable approach for the treatment of oxidative stress-related disease. Hydrogen 
is a promising gaseous agent that has come to the forefront of therapeutic research over the last few years. It can selectively reduce cytotoxic ROS and exert organ-protective effects through regulation of oxidative stress and inflammation. Hydrogen is so mild that it does not disturb metabolic oxidation-reduction reactions or disrupt the ROS involved in cell signaling. As highlighted earlier, the efficiency and safety of hydrogen in ameliorating ocular pathologies have been verified by a series of experiments. The pioneering attempts reviewed in the present work ignite the hope of discovering a novel, easy, and universal therapeutic tool against ocular diseases. Future hydrogen therapy should endeavor to establish well-defined administration guidelines and determine precise indications for clinical practice.

\section{Acknowledgment}

This work is supported by the National Key Basic Research Program of China (No 2013CB967001).

\section{Disclosure}

The authors report no conflicts of interest in this work.

\section{References}

1. Wallace DC. A mitochondrial paradigm of metabolic and degenerative diseases, aging, and cancer: a dawn for evolutionary medicine. Annu Rev Genet. 2005;39:359-407.

2. Reddy PH. Amyloid precursor protein-mediated free radicals and oxidative damage: implications for the development and progression of Alzheimer's disease. J Neurochem. 2006;96:1-13.

3. Ohta SA. Multi-functional organelle mitochondrion is involved in cell death, proliferation and disease. Curr Med Chem. 2003;10:2485-2494.

4. Fang J, Seki T, Maeda H. Therapeutic strategies by modulating oxygen stress in cancer and inflammation. Adv Drug Deliv Rev. 2009;61: 290-302.

5. Davies KJ. Oxidative stress: the paradox of aerobic life. Biochem Soc Symp. 1995;61:1-31.

6. Sundaresan M, Yu ZX, Ferrans VJ, Irani K, Finkel T. Requirement for generation of $\mathrm{H}_{2} \mathrm{O}_{2}$ for platelet-derived growth factor signal transduction. Science. 1995;270:296-299.

7. Valko M, Leibfritz D, Moncol J, Cronin MT, Mazur M, Telser J. Free radicals and antioxidants in normal physiological functions and human disease. Int J Biochem Cell Biol. 2007;39:44-84.

8. Ichihara M, Sobue S, Ito M, Ito M, Hirayama M, Ohno K. Beneficial biological effects and the underlying mechanisms of molecular hydrogen comprehensive review of 321 original articles. Med Gas Res. 2015; $5: 12$.

9. Vijayalaxmi, Reiter RJ, Tan DX, Herman TS, Thomas CR Jr. Melatonin as a radioprotective agent: a review. Int J Radiat Oncol Biol Phys. 2004; 59:639-653.

10. Gilgun-Sherki Y, Rosenbaum Z, Melamed E, et al. Antioxidant therapy in acute central nervous system injury: current state. Pharmacol Rev. 2002;54:271-284.

11. Tator $\mathrm{CH}$. Review of treatment trials in human spinal cord injury: issues, difficulties, and recommendations. Neurosurgery. 2006;59:957-982.

12. Dole M, Wilson FR, Fife WP. Hyperbaric hydrogen therapy: a possible treatment for cancer. Science. 1975;190(4210):152-154.

13. Ohsawa I, Ishikawa M, Takahashi K, et al. Hydrogen acts as a therapeutic antioxidant by selectively reducing cytotoxic oxygen radicals. Nat Med. 2007;13(6):688-694.
14. Nakao A, Toyoda Y, Sharma P, Evans M, Guthrie N. Effectiveness of hydrogen rich water on antioxidant status of subjects with potential metabolic syndrome - an open label pilot study. J Clin Biochem Nutr. 2010; 46:140-149.

15. Liu Q, Shen WF, Sun HY, et al. Hydrogen-rich saline protects against liver injury in rats with obstructive jaundice. Liver Int. 2010;30: 958-968.

16. Fukuda K, Asoh S, Ishikawa M, Yamamoto Y, Ohsawa I, Ohta S. Inhalation of hydrogen gas suppresses hepatic injury caused by ischemia/ reperfusion through reducing oxidative stress. Biochem Biophys Res Commun. 2007;361:670-674.

17. Sun Q, Kang Z, Cai J, et al. Hydrogen-rich saline protects myocardium against ischemia/reperfusion injury in rats. Exp Biol Med (Maywood). 2009;234:1212-1219.

18. Nakao A, Kaczorowski DJ, Wang Y, et al. Amelioration of rat cardiac cold ischemia/reperfusion injury with inhaled hydrogen or carbon monoxide, or both. J Heart Lung Transplant. 2010;29:544-553.

19. Cardinal JS, Zhan J, Wang Y, et al. Oral hydrogen water prevents chronic allograft nephropathy in rats. Kidney Int. 2010;77:101-109.

20. Li J, Wang C, Zhang JH, Cai JM, Cao YP, Sun XJ. Hydrogen-rich saline improves memory function in a rat model of amyloid-beta-induced Alzheimer's disease by reduction of oxidative stress. Brain Res. 2010; 1328:152-161.

21. Chen C, Chen Q, Mao Y, et al. Hydrogen-rich saline protects against spinal cord injury in rats. Neurochem Res. 2010;35:1111-1118.

22. Fu Y, Ito M, Fujita $Y$, et al. Molecular hydrogen is protective against 6-hydroxydopamine-induced nigrostriatal degeneration in a rat model of Parkinson. Neurosci Lett. 2009;453(2):81-85.

23. Yang CX, Yan H, Ding TB. Hydrogen saline prevents selenite-induced cataract in rats. Mol Vis. 2013;19:1684-1693.

24. Hong Y, Chen S, Zhang JM. Hydrogen as a selective antioxidant: A review of clinical and experimental studies. J Int Med Res. 2010;38: 1893-1903.

25. Ono H, Nishijima Y, Adachi N, et al. Improved brain MRI indices in the acute brain stem infarct sites treated with hydroxyl radical scavengers, Edaravone and hydrogen, as compared to Edaravone alone. A non-controlled study. Med Gas Res. 2011;1(1):12.

26. Gharib B, Hanna S, Abdallahi OM, Lepidi H, Gardette B, De Reggi M. Anti-inflammatory properties of molecular hydrogen: investigation on parasite-induced liver inflammation. C R Acad Sci III. 2001;324(8): 719-724.

27. Kajiya M, Silva MJ, Sato K, Ouhara K, Kawai T. Hydrogen mediates suppression of colon inflammation induced by dextran sodium sulfate. Biochem Biophys Res Commun. 2009;386:11-15.

28. Liu GD, Zhang H, Wang L, Han Q, Zhou SF, Liu P. Molecular hydrogen regulates the expression of miR-9, miR-21 and miR-199 in LPS-activated retinal microglia cells. Int J Ophthalmol. 2013;6(3): 280-285.

29. Ge Y, Wu F, Sun X, et al. Intrathecal infusion of hydrogen-rich normal saline attenuates neuropathic pain via inhibition of activation of spinal astrocytes and microglia in rats. PLoS One. 2014;9(5):e97436.

30. Abraini JH, Gardette-Chauffour MC, Martinez E, et al. Psychophysiological reactions in humans during an open sea dive to $500 \mathrm{~m}$ with a hydrogen-helium-oxygen mixture. J Appl Physiol. 1994;76(3): 1113-1118.

31. Roberts BJ, Fife WP, Corbett TH, Schabel FM Jr. Response of five established solid transplantable mouse tumors and one mouse leukemia to hyperbaric hydrogen. Cancer Treat Rep. 1978;62(7):1077-1079.

32. Buchholz BM, Kaczorowski DJ, Sugimoto R, et al. Hydrogen inhalation ameliorates oxidative stress in transplantation induced intestinal graft injury. Am J Transplant. 2008;8:2015-2024.

33. Zhou L, Wang X, Xue W, et al. Beneficial effects of hydrogen-rich saline against spinal cord ischemia-reperfusion injury in rabbits. Brain Res. 2013; 1517:150-160.

34. Zhang L, Shu R, Wang H, et al. Hydrogen-rich saline prevents remifentanil-induced hyperalgesia and inhibits MnSOD nitration via regulation of NR2B-containing NMDA receptor in rats. Neuroscience. 2014;280: 171-180. 
35. Lin Y, Kashio A, Sakamoto T, Suzukawa K, Kakigi A, Yamasoba T. Hydrogen in drinking water attenuates noise-induced hearing loss in guinea pigs. Neurosci Lett. 2011;487(1):12-16.

36. Terawaki H, Hayashi Y, Zhu WJ, et al. Transperitoneal administration of dissolved hydrogen for peritoneal dialysis patients: a novel approach to suppress oxidative stress in the peritoneal cavity. Med Gas Res. 2013; 3(1): 14 .

37. Takeuchi S, Mori K, Arimoto H, et al. Effects of intravenous infusion of hydrogen-rich fluid combined with intra-cisternal infusion of magnesium sulfate in severe aneurysmal subarachnoid hemorrhage: study protocol for a randomized controlled trial. BMC Neurol. 2014; $14: 176$.

38. Wei L, Ge L, Qin S, et al. Hydrogen-rich saline protects retina against glutamate-induced excitotoxic injury in guinea pig. Exp Eye Res. 2012; 94(1):117-127.

39. Oharazawa H, Igarashi T, Yokota T, et al. Protection of the retina by rapid diffusion of hydrogen: administration of hydrogen-loaded eye drops in retinal ischemia-reperfusion injury. Invest Ophthalmol Vis Sci. 2010;51(1):487-492.

40. Sun XJ, Zhang JH. Hydrogen - an endogenous antioxidant in the body. Acad J Sec Mil Med Univ. 2008;29(3):233-235.

41. Noell WK. Aspects of experimental and hereditary degeneration. In: Graymore C, editor. Biochemistry of the Retina. London: Academic Press; 1965:51-72.

42. Heckenlively JR. Retinitis Pigmentosa. Philadelphia: JB Lippincott; 1988.

43. Cruickshanks KJ, Klein R, Klein BE, Nondahl DM. Sunlight and age-related macular degeneration. The Beaver Dam Eye Study. Arch Ophthalmol. 1993;111:514-518.

44. Fletcher AE, Bentham GC, Agnew M, et al. Sunlight exposure, antioxidants, and age-related macular degeneration. Arch Ophthalmol. 2008;126:1396-1403.

45. Hartong DT, Berson EL, Dryja TP. Retinitis pigmentosa. Lancet. 2006 ; 368:1795-1809

46. Tan JS, Wang JJ, Flood V, Rochtchina E, Smith W, Mitchell P. Dietary antioxidants and the long-term incidence of age-related macular degeneration: the Blue Mountains Eye Study. Ophthalmology. 2008; 115:334-341.

47. Organisciak DT, Vaughan DK. Retinal light damage: mechanisms and protection. Prog Retin Eye Res. 2010;29:113-134.

48. Organisciak DT, Wang HM, Li ZY, Tso MO. The protective effect of ascorbate in retinal light damage of rats. Invest Ophthalmol Vis Sci. 1985; $26: 1580-1588$

49. Organisciak DT, Darrow RM, Jiang YI, Marak GE, Blanks JC. Protection by dimethylthiourea against retinal light damage in rats. Invest Ophthalmol Vis Sci. 1992;33:1599-1609.

50. Feng M, Wang XH, Yang XB, Xiao Q, Jiang FG. Protective effect of saturated hydrogen saline against blue light-induced retinal damage in rats. Int J Ophthalmol. 2012;5(2):151-157.

51. Tian L, Zhang L, Xia F, An J, Sugita Y, Zhang Z. Hydrogen-rich saline ameliorates the retina against light-induced damage in rats. Med Gas Res. 2013;3(1):19.

52. Barber AJ. A new view of diabetic retinopathy: a neurodegenerative disease of the eye. Prog Neuropsychopharmacol Biol Psychiatry. 2003; 27:283-290.

53. Forbes JM, Coughlan MT, Cooper ME. Oxidative stress as a major culprit in kidney disease in diabetes. Diabetes. 2008;57:1446-1454.

54. Zheng Z, Chen H, Ke G, et al. Protective effect of perindopril on diabetic retinopathy is associated with decreased vascular endothelial growth factor-to-pigment epithelium-derived factor ratio: involvement of a mitochondria-reactive oxygen species pathway. Diabetes. 2009;58: 954-964.

55. Rivero A, Mora C, Muros M, García J, Herrera H, Navarro-González JF. Pathogenic perspectives for the role of inflammation in diabetic nephropathy. Clin Sci. 2009;116:479-492.

56. Xiao X, Cai J, Xu J, et al. Protective effects of hydrogen saline on diabetic retinopathy in a streptozotocin-induced diabetic rat model. J Ocul Pharmacol Ther. 2012;28(1):76-82.
57. Feng Y, Wang $\mathrm{R}, \mathrm{Xu}$ J, et al. Hydrogen-rich saline prevents early neurovascular dysfunction resulting from inhibition of oxidative stress in STZ-diabetic rats. Curr Eye Res. 2013;38(3):396-404.

58. Dong A, Xie B, Shen J, et al. Oxidative stress promotes ocular neovascularization. J Cell Physiol. 2009;219:544-552.

59. Guma M, Rius J, Duong-Polk KX, Haddad GG, Lindsey JD, Karin M. Genetic and pharmacological inhibition of JNK ameliorates hypoxiainduced retinopathy through interference with VEGF expression. PNAS. 2009; 106:8760-8765.

60. Huang L, Zhao S, Zhang JH, Sun X. Hydrogen saline treatment attenuates hyperoxia-induced retinopathy by inhibition of oxidative stress and reduction of VEGF expression. Ophthalmic Res. 2012;47(3):122-127.

61. Kajiyama S, Hasegawa G, Asano M, et al. Supplementation of hydrogenrich water improves lipid and glucose metabolism in patients with type 2 diabetes or impaired glucose tolerance. Nutr Res. 2008;28(3): 137-143.

62. Bien A, Seidenbecher CI, Bockers TM, Sabel BA, Kreutz MR. Apoptotic versus necrotic characteristics of retinal ganglion cell death after partial optic nerve injury. J Neurotrauma. 1999;16:153-163.

63. Levkovitch-Verbin H, Harris-Cerruti C, Groner Y, Wheeler LA, Schwartz M, Yoles E. RGC death in mice after optic nerve crush injury: oxidative stress and neuroprotection. Invest Ophthalmol Vis Sci. 2000; 41:4169-4174.

64. Sun JC, Xu T, Zuo Q, et al. Hydrogen-rich saline promotes survival of retinal ganglion cells in a rat model of optic nerve crush. PLoS One. 2014;9(6):e99299.

65. Bockelbrink A, Roll S, Ruether K, Rasch A, Greiner W, Willich SN. Cataract surgery and the development or progression of age-related macular degeneration: a systematic review. Surv Ophthalmol. 2008;53:359-367.

66. Toda J, Kato S, Oshika T, Sugita G. Posterior capsule opacification after combined cataract surgery and vitrectomy. $J$ Cataract Refract Surg. 2007;33:104-107.

67. Beebe DC, Holekamp NM, Shui YB. Oxidative damage and the prevention of age-related cataracts. Ophthalmic Res. 2010;44:155-165.

68. Chang JH, Gabison EE, Kato T, Azar DT. Corneal neovascularization. Curr Opin Ophthalmol. 2001;12:242-249.

69. Lee P, Wang CC, Adamis AP. Ocular neovascularization: an epidemiologic review. Surv Ophthalmol. 1998;43:245-269.

70. Baldwin AS Jr. The NF-kappa B and I kappa B proteins: new discoveries and insights. Annu Rev Immunol. 1996;14:649-683.

71. Kubota M, Shimmura S, Kubota S, et al. Hydrogen and N-acetyl-Lcysteine rescue oxidative stress-induced angiogenesis in a mouse cornea alkali-burn model. Invest Ophthalmol Vis Sci. 2011;52(1):427-433.

72. Hughes WF. Quantitation of ischemic damage in the rat retina. Exp Eye Res. 1991;53:573-582.

73. Kuroiwa S, Katai N, Shibuki H, et al. Expression of cell cycle-related genes in dying cells in retinal ischemic injury. Invest Ophthalmol Vis Sci. 1998;39:610-617

74. Tezel G. Oxidative stress in glaucomatous neurodegeneration: mechanisms and consequences. Prog Retinal Eye Res. 2006;25:490-513.

75. Shibuki H, Katai N, Yodoi J, Uchida K, Yoshimura N. Lipid peroxidation and peroxynitrite in retinal ischemia-reperfusion injury. Invest Ophthalmol Vis Sci. 2000;41:3607-3614.

76. Hirooka K, Miyamoto O, Jinming P, et al. Nueroprotective effects of $\mathrm{D}$-allose against retinal ischemia-reperfusion injury. Invest Ophthalmol Vis Sci. 2006;47:1653-1657.

77. Liu H, Hua N, Xie K, Zhao T, Yu Y. Hydrogen-rich saline reduces cell death through inhibition of DNA oxidative stress and overactivation of poly (ADPribose) polymerase-1 in retinal ischemia-reperfusion injury. Mol Med Rep. 2015;12(2):2495-2502.

78. Guo L, Salt TE, Maass A, et al. Assessment of neuroprotective effects of glutamate modulation on glaucoma-related retinal ganglion cell apoptosis in vivo. Invest. Invest Ophthalmol Vis Sci. 2006;47:626-633. 


\section{Publish your work in this journal}

Therapeutics and Clinical Risk Management is an international, peerreviewed journal of clinical therapeutics and risk management, focusing on concise rapid reporting of clinical studies in all therapeutic areas, outcomes, safety, and programs for the effective, safe, and sustained use of medicines. This journal is indexed on PubMed Central, CAS,

EMBase, Scopus and the Elsevier Bibliographic databases. The manuscript management system is completely online and includes a very quick and fair peer-review system, which is all easy to use. Visit http://www.dovepress.com/testimonials.php to read real quotes from published authors.

Submit your manuscript here: http://www.dovepress.com/therapeutics-and-clinical-risk-management-journal 